

\title{
Heights of roots of polynomials with odd coefficients
}

\author{
par J. GARZA, M. I. M. ISHAK, M. J. MOSSINGHOFF, \\ C. G. PINNER et B. WILES
}

RÉSUMÉ. Soit $\alpha$ un zero d'un polynôme de degré $n$ à coefficients impairs qui n'est pas une racine de l'unité. Nous montrons que la hauteur de $\alpha$ satisfait

$$
h(\alpha) \geq \frac{0.4278}{n+1} .
$$

Plus généralement, nous obtenons des bornes dans le cas où chaque coefficient est congru à 1 modulo $m$, avec $m \geq 2$.

ABSTRACT. Let $\alpha$ be a zero of a polynomial of degree $n$ with odd coefficients, with $\alpha$ not a root of unity. We show that the height of $\alpha$ satisfies

$$
h(\alpha) \geq \frac{0.4278}{n+1} .
$$

More generally, we obtain bounds when the coefficients are all congruent to 1 modulo $m$ for some $m \geq 2$.

\section{Introduction}

We recall the Mahler measure $M(f)$ of a polynomial $f=a \prod_{i=1}^{d}\left(x-\alpha_{i}\right)$ in $\mathbb{C}[x]$ :

$$
M(f)=|a| \prod_{i=1}^{d} \max \left\{1,\left|\alpha_{i}\right|\right\} .
$$

For a nonzero algebraic number $\alpha$ of degree $d$, one defines the absolute logarithmic height $h(\alpha)$ of $\alpha$ to be

$$
h(\alpha)=\frac{1}{d} \log M(F),
$$

where $F$ is an irreducible polynomial in $\mathbb{Z}[x]$ with $F(\alpha)=0$. That is, $\log M(f)$ represents the sum of the heights of the nonzero roots of $f$ (with multiplicity) whenever $f$ is primitive in $\mathbb{Z}[x]$.

Manuscrit reçu le 24 mai 2009, révisé le 15 décembre 2009.

The research of the third author was supported in part by NSA grant H98230-08-1-0052.

Mots clefs. Heights, Mahler measure, Lehmer's problem.

Classification math.. 11R09, 11C08, 11R06. 
For an integer $m \geq 2$, let $D_{m}$ denote the set of integer polynomials whose coefficients $a_{i}$ all satisfy $a_{i} \equiv 1(\bmod m)$. For a polynomial of degree $n$ in $D_{m}$ with no cyclotomic factors, Borwein, Dobrowolski, and Mossinghoff [1] proved that

$$
\log M(f) \geq c_{m} \frac{n}{n+1},
$$

with $c_{2}=\frac{1}{4} \log 5=0.402359 \ldots, c_{3}=0.459003$, and $c_{m}=\log \left(\sqrt{m^{2}+1} / 2\right)$ for $m>3$. These constants were improved in [2] to obtain $c_{2}=0.416230 \ldots$, general bounds of strength

$$
c_{m}= \begin{cases}\log (m / 2)+(3-\log 3) / 2 m^{2}+O\left(1 / m^{4}\right) & \text { if } m \geq 3 \text { odd } \\ \log (m / 2)+(4-\log 4) / m^{2}+O\left(1 / m^{4}\right) & \text { if } m \geq 4 \text { even, }\end{cases}
$$

and particular values $c_{3}=0.501026 \ldots, c_{4}=0.832461 \ldots, c_{5}=0.952869$, $c_{6}=1.165884, c_{7}=1.271775, c_{8}=1.425369, c_{9}=1.515669, c_{10}=$ 1.634836 , and $c_{11}=1.712539$.

We show here how to more straightforwardly obtain bounds of the form

$$
h(\alpha) \geq \frac{c_{m}}{n+1}
$$

when $\alpha$ is a zero of a polynomial $f$ in $D_{m}$ of degree $n$, but not a $2(n+1)$ st root of unity. Of course then

$$
\log M(f) \geq c_{m} \frac{d}{n+1}
$$

where $d$ is the degree of the noncyclotomic part of $f$ (the type of bound obtained in Theorem 2.2 of [2]).

Theorem 1.1. If $\alpha$ is a zero of a polynomial $f$ in $D_{m}$ of degree $n$ and $\alpha$ is not a $2(n+1)$ st root of unity (not an $(n+1)$ st if $m \geq 3)$, then (1.1) holds with

$$
c_{2}=0.427800
$$

and

$$
c_{m}=\log \left(\frac{m}{2}\right)+\frac{2.947486-\delta / 2}{m^{2}}+O\left(\frac{1}{m^{4}}\right)
$$

where

$$
\delta= \begin{cases}1 & \text { if } m \geq 3 \text { odd } \\ 0 & \text { if } m \geq 4 \text { even }\end{cases}
$$

For small $m \geq 3$ we show the following improvements: $c_{3}=0.620362$, $c_{4}=0.855600, c_{5}=1.016628, c_{6}=1.179916, c_{7}=1.307083, c_{8}=1.434141$, $c_{9}=1.538934, c_{10}=1.640027$, and $c_{11}=1.728890$. 
We note the easily obtained (if asymptotically less precise) bound

$$
c_{m}= \begin{cases}\frac{1}{2} \log \left(\frac{m^{2}+3}{4}\right) & \text { if } m \geq 3 \text { odd } \\ \frac{1}{2} \log \left(\frac{m^{2}+4}{4}\right) & \text { if } m \geq 4 \text { even }\end{cases}
$$

(the even case having already been obtained and improved in [2]). We remark also that the same computation that yields the value of $c_{3}$ in Theorem 1.1 immediately produces the lower bound

$$
h(\alpha) \geq 0.155090
$$

for abelian $\alpha$ (see [3]).

Our second main result shows that the optimal $c_{m}$ in (1.1) certainly satisfies $c_{m}=\log m+O(1)$.

Theorem 1.2. If (1.1) holds for any non-root of unity $\alpha$ that is a zero of a polynomial $f$ in $D_{m}$ of degree $n$, then

$$
c_{2} \leq \log \left(\frac{1+\sqrt{5}}{2}\right)=0.481211 \ldots
$$

(even if we further restrict to Littlewood polynomials),

$$
\begin{gathered}
c_{3} \leq \log 2=0.693147 \ldots, \\
c_{4} \leq \log (1+\sqrt{2})=0.881373 \ldots,
\end{gathered}
$$

and

$$
c_{6} \leq \log \left(\frac{3+\sqrt{13}}{2}\right)=1.194763 \ldots .
$$

Further, for general $m \geq 3$,

$$
c_{m} \leq \log (m-1)
$$

It is not clear what the optimal constant $C_{1}$ should be in a bound of the form $c_{m}=\log m-C_{1}+o(1)$.

\section{Preliminaries}

Suppose that $\alpha$ lies in an algebraic number field $k$, and $V_{k}$ is a complete set of absolute values ||$_{v}$ on $k$, normalised so that $|x|_{v}=\|x\|_{v}^{d_{v} / d}$ where $d=[k: \mathbb{Q}], d_{v}=\left[k_{v}: \mathbb{Q}_{v}\right]$, and $\|x\|_{v}$ coincides with the usual absolute value or $p$-adic absolute value on $\mathbb{Q}$. Then

$$
h(\alpha)=\log H(\alpha), \quad H(\alpha)=\prod_{v \in V_{k}} \max \left\{1,|\alpha|_{v}\right\} .
$$

The normalisations ensure that this does not depend upon $k$. 
Lemma 2.1. For $t=1$, or $t>1$ and $k \leq 4 t /(t-1)^{2}$,

$$
\sup _{|z|=1}\left|(z-1)^{k}(z+t)\right|=\frac{(t+1)^{k+1}}{(k+1)^{\frac{1}{2}(k+1)}}\left(\frac{k}{t}\right)^{\frac{1}{2} k}
$$

achieved at $z=-\frac{\left(\left(t^{2}+1\right) k-2 t\right)}{2 t(k+1)} \pm \frac{(t+1) \sqrt{k\left(4 t-(t-1)^{2} k\right)}}{2 t(k+1)}$. For $t>1$ and $k \geq$ $4 t /(t-1)^{2}$, the supremum is $2^{k}(t-1)$, achieved at $z=-1$.

Proof. Writing $z=e^{i \theta}, u=\cos \theta$, it is readily checked that

$$
\left|(z-1)^{k}(z+t)\right|^{2}=2^{k}(1-u)^{k}\left(\left(t^{2}+1\right)+2 t u\right)
$$

is maximised for $-1 \leq u \leq 1$ at $u=-\frac{\left(\left(t^{2}+1\right) k-2 t\right)}{2 t(k+1)}$ while this is at least -1 (and at $u=-1$ when $k>4 t /(t-1)^{2}$ ).

Define the polynomials

$$
g_{1}(z)=\frac{1}{2}(m-\delta) z+\frac{1}{2}(m+\delta), \quad \delta= \begin{cases}1 & \text { if } m \text { is odd } \\ 0 & \text { if } m \text { is even }\end{cases}
$$

and

$$
g_{2}(z)=\frac{1}{4}\left(m^{2}+(4-\delta)\right) z^{2}+\frac{1}{2}\left(m^{2}-(4-\delta)\right) z+\frac{1}{4}\left(m^{2}+(4-\delta)\right) .
$$

Lemma 2.2. If $m \geq 3$ is odd then $g_{1}\left(z^{n}\right)$ is irreducible in $\mathbb{Z}[z]$ for all $n$ in $\mathbb{N}$. Further, if $m \geq 4$ is odd with $3 \nmid m$ or even with $4 \mid m$ then $g_{2}\left(z^{n}\right)$ is irreducible in $\mathbb{Z}[z]$ for all $n$ in $\mathbb{N}$.

Proof. If $m=2 k+1$, is odd then by Capelli's Theorem $g_{1}\left(z^{n}\right)=k z^{n}+(k+1)$ is irreducible unless $(k+1) / k$ is a prime power in $\mathbb{Q}$, but plainly $k+1=a^{p}$, $k=b^{p}$ has no positive integer solutions $a, b$.

Observe that if $g_{2}(\beta)=0$ then

$$
\beta=\frac{-\frac{1}{2}\left(m^{2}-(4-\delta)\right) \pm m \sqrt{(4-\delta)} i}{\frac{1}{2}\left(m^{2}+(4-\delta)\right)}
$$

is complex, lying on the unit circle. Moreover, if $m$ is odd and $3 \nmid m$, or if $4 \mid m$, then

$$
\operatorname{gcd}\left(\frac{1}{4}\left(m^{2}+(4-\delta)\right), \frac{1}{2}\left(m^{2}-(4-\delta)\right)\right)=1,
$$

$g_{2}(z)$ is irreducible in $\mathbb{Z}[z]$, and

$$
h(\beta)=\frac{1}{2} \log \left(\frac{m^{2}+(4-\delta)}{4}\right) .
$$


Notice that if $m \geq 2$ is odd with $3 \mid m$, or if $2 \| m$, then we need to first factor out a common 3 or 2 and

$$
h(\beta)=\frac{1}{2} \log \left(\frac{m^{2}+3}{12}\right) \text { or } \frac{1}{2} \log \left(\frac{m^{2}+4}{8}\right) .
$$

Suppose then that $(m, 6)=1$ or $4 \mid m$, and $g_{2}\left(z^{n}\right)$ has a nontrivial factor,

$$
r(z)=\sum_{i=0}^{d} a_{i} z^{i} \in \mathbb{Z}[z], \quad a_{d} \neq 0, \quad 0<d<2 n .
$$

If $\alpha$ is a root of $r(z)$, then

$$
\frac{\log \left|a_{d}\right|}{d}=h(\alpha)=\frac{1}{n} h(\beta)
$$

and

$$
\frac{m^{2}+(4-\delta)}{4}=\left|a_{d}\right|^{2 n / d}=y^{p}
$$

for some $y$ in $\mathbb{N}$ and prime $p \mid 2 n / \operatorname{gcd}(2 n, d)$. For $m=4 l$, this reduces to $l^{2}+1=y^{p}$, a special case of Catalan's equation shown to have no solution by Lebesgue [4]. For odd $m=2 l+1$, this reduces to $l^{2}+l+1=y^{p}$, which was shown by Nagell [6] and Ljunggren [5] to have only the solution $p=3$, $y=7, l=18$. This just leaves the case $m=37$, in which case

$$
g_{2}\left(z^{n}\right)=7^{3}\left(z^{n}-\beta\right)\left(z^{n}-\beta^{-1}\right), \quad \beta=\frac{1}{2}(1-\sqrt{3} i)\left(\frac{2+\sqrt{3} i}{2-\sqrt{3} i}\right)^{3} .
$$

Plainly then $g_{2}\left(z^{n}\right)$ is irreducible in $\mathbb{Z}[z]$ unless $\left(z^{n}-\beta\right)$ is reducible in $\mathbb{Q}(\sqrt{3} i)[z]$. But by Capelli's Theorem this would require $\beta=A^{p}$ or $-4 \mu^{4}$ for some prime $p$ and $A$ or $\mu$ in $\mathbb{Q}(\sqrt{3} i)$. Considering prime factorizations in the integers of $\mathbb{Q}(\sqrt{3} i)$, the only possibility would be $p=3$, but $\frac{1}{2}(1-\sqrt{3} i)$ cannot be a cube in $\mathbb{Q}(\sqrt{3} i)$ (which contains the sixth but not the eighteenth roots of unity).

\section{Proof of Theorem 1.1}

If $f$ is in $D_{m}$, then $f(x)=\frac{x^{n+1}-1}{x-1}+m r(x)$ for some $r$ of degree at most $n$ in $\mathbb{Z}[x]$. Hence for $v \nmid \infty$, writing $\beta=\alpha^{n+1}$,

$$
|\beta-1|_{v}=|m(\alpha-1) r(\alpha)|_{v} \leq|m|_{v} \max \left\{1,|\beta|_{v}\right\}
$$

For $m=2$ we take

$$
\begin{aligned}
g(z)= & (z-1)^{k}(z+1)^{l}\left(5 z^{2}+6 z+5\right)^{t}\left(29 z^{4}+60 z^{3}+78 z^{2}+60 z+29\right)^{w} \\
& \cdot\left(3 z^{2}+2 z+3\right)^{c}\left(33 z^{4}+60 z^{3}+70 z^{2}+60 z+33\right)^{e} \\
& \cdot\left(169 z^{6}+490 z^{5}+871 z^{4}+1036 z^{3}+871 z^{2}+490 z+169\right)^{s} .
\end{aligned}
$$


Thus for $v \nmid \infty$,

$$
\begin{aligned}
& |\beta-1|_{v} \leq|2|_{v} \max \left\{1,|\beta|_{v}\right\} \\
& |\beta+1|_{v}=|\beta-1+2|_{v} \leq|2|_{v} \max \left\{1,|\beta|_{v}\right\}
\end{aligned}
$$

and

$$
\left|\beta^{2}-1\right|_{v} \leq|2|_{v}^{2} \max \left\{1,|\beta|_{v}\right\}^{2}
$$

giving

$$
\begin{gathered}
\left|\beta^{2}+1\right|_{v}=\left|\beta^{2}-1+2\right|_{v} \leq|2|_{v} \max \left\{1,|\beta|_{v}\right\}^{2}, \\
\left|5 \beta^{4}+6 \beta^{2}+5\right|_{v}=\left|5\left(\beta^{2}-1\right)^{2}+16 \beta^{2}\right|_{v} \leq|2|_{v}^{4} \max \left\{1,|\beta|_{v}\right\}^{4}, \\
\left|3 \beta^{4}+2 \beta^{2}+3\right|_{v}=\left|3\left(\beta^{2}-1\right)^{2}+8 \beta^{2}\right|_{v} \leq|2|_{v}^{3} \max \left\{1,|\beta|_{v}\right\}^{4},
\end{gathered}
$$

and for integers $A, B, C$, and $D$,

$$
\left|A\left(\beta^{2}-1\right)^{4}+B 2^{4} \beta^{2}\left(\beta^{2}-1\right)^{2}+C 2^{8} \beta^{4}\right|_{v} \leq|2|_{v}^{8} \max \left\{1,|\beta|_{v}\right\}^{8},
$$

and

$$
\begin{aligned}
\left|A\left(\beta^{2}-1\right)^{6}+B 4^{2} \beta^{2}\left(\beta^{2}-1\right)^{4}+C 4^{4} \beta^{4}\left(\beta^{2}-1\right)^{2}+D 4^{6} \beta^{6}\right|_{v} \\
\leq|2|_{v}^{12} \max \left\{1,|\beta|_{v}\right\}^{12} .
\end{aligned}
$$

The two quartic factors in $g(z)$ correspond to $(A, B, C)=(29,11,1)$ and $(33,12,1)$ in $(3.3)$, and the sextic to $(A, B, C, D)=(169,94,17,1)$ in $(3.4)$. Hence we have

$$
\left|g\left(\beta^{2}\right)\right|_{v} \leq|2|_{v}^{2 k+l+4 t+8 w+3 c+8 e+12 s} \max \left\{1,|\beta|_{v}\right\}^{2 \operatorname{deg} g}
$$

for $v \nmid \infty$.

For $v \mid \infty$ and $|\beta|_{v}>1$, we observe that $\left|g\left(\beta^{2}\right)\right|_{v}=|\beta|_{v}^{2 \operatorname{deg} g}\left|g\left(\beta^{-2}\right)\right|_{v}$ with $\left|\beta^{-2}\right|_{v}<1$. Hence for $v \mid \infty$,

$$
\begin{aligned}
\left|g\left(\beta^{2}\right)\right|_{v} & \leq \max \left\{1,|\beta|_{v}\right\}^{2 \operatorname{deg} g}\left(\sup _{|z| \leq 1}|g(z)|\right)^{d_{v} / d} \\
& =\max \left\{1,|\beta|_{v}\right\}^{2 \operatorname{deg} g} \sqrt{M}^{d_{v} / d}
\end{aligned}
$$

where, writing $z=e^{i t}$ and $u=\cos t$,

$$
M=\sup _{|z|=1}|g(z)|^{2}=2^{k+l+2 t+4 w+2 c+4 e+6 s} L,
$$

with

$$
\begin{gathered}
L=\sup _{-1 \leq u \leq 1}(1-u)^{k}(1+u)^{l}(5 u+3)^{2 t}\left(29 u^{2}+30 u+5\right)^{2 w}(3 u+1)^{2 c} \\
\cdot\left(33 u^{2}+30 u+1\right)^{2 e}\left(169 u^{3}+245 u^{2}+91 u+7\right)^{2 s}
\end{gathered}
$$


We need to justify that $g\left(\beta^{2}\right) \neq 0$. By assumption $\beta^{2} \neq 1$, and from $(3.2)$ plainly $\beta^{2} \neq-1$. Observe also that

$$
\begin{gathered}
5 z^{4(n+1)}+6 z^{2(n+1)}+5, \\
3 z^{4(n+1)}+2 z^{2(n+1)}+3, \\
29 z^{8(n+1)}+60 z^{6(n+1)}+78 z^{4(n+1)}+60 z^{2(n+1)}+29, \\
33 z^{8(n+1)}+60 z^{6(n+1)}+70 z^{4(n+1)}+60 z^{2(n+1)}+33,
\end{gathered}
$$

and the factors

$$
\begin{aligned}
& 13 z^{6(n+1)}+2 z^{5(n+1)}+19 z^{4(n+1)}-4 z^{3(n+1)}+19 z^{2(n+1)}+2 z^{(n+1)}+13, \\
& 13 z^{6(n+1)}-2 z^{5(n+1)}+19 z^{4(n+1)}+4 z^{3(n+1)}+19 z^{2(n+1)}-2 z^{(n+1)}+13
\end{aligned}
$$

of

$$
\begin{aligned}
& 169 z^{12(n+1)}+490 z^{10(n+1)}+871 z^{8(n+1)}+1036 z^{6(n+1)}+871 z^{4(n+1)} \\
& +490 z^{2(n+1)}+169
\end{aligned}
$$

are all irreducible (each of their roots lies on the unit circle with the same nontrivial height, so the lead coefficients of each factor would need to contain all the primes in the original lead coefficient). Since $\alpha$ has degree at most $n$, the remaining factors cannot vanish. Thus, by the product formula,

$$
1=\prod_{v}\left|g\left(\beta^{2}\right)\right|_{v} \leq H(\beta)^{2 \operatorname{deg} g} 2^{-(2 k+l+4 t+8 w+3 c+8 e+12 s)} \sqrt{M},
$$

and

$$
h(\beta) \geq \frac{\log \left(2^{3 k+l+6 t+12 w+4 c+12 e+18 s} / L\right)}{4(k+l+2 t+4 w+2 c+4 e+6 s)} .
$$

The choice $(k, l, t, w, c, e, s)=(3977,780,328,96,24,16,16)$ and numerical computation of $L$ gives the lower bound $h(\beta) \geq 0.4278003111 \ldots$ claimed.

For $m=4$, taking $g(\beta)$ in place of $g\left(\beta^{2}\right)$ immediately produces $h(\beta) \geq$ $2 \cdot 0.4278003111 \ldots=0.8556006223 \ldots$

For general $m \geq 3$, we take

$$
g(z)=\prod_{i=0}^{I} g_{i}(z)^{s_{i}}
$$

with $I=2, g_{0}(z)=z-1$, and $g_{1}(z)$ and $g_{2}(z)$ as in (2.1) and (2.2). For $v \nmid \infty$ we have

$$
\begin{gathered}
\left|g_{1}(\beta)\right|_{v}=\left|\frac{1}{2}(m-\delta)(\beta-1)+m\right|_{v} \leq|m|_{v} \max \left\{1,|\beta|_{v}\right\} \\
\left|g_{2}(\beta)\right|_{v}=\left|\frac{1}{4}\left(m^{2}+(4-\delta)\right)(\beta-1)^{2}+m^{2} \beta\right|_{v} \leq|m|_{v}^{2} \max \left\{1,|\beta|_{v}\right\}^{2}
\end{gathered}
$$


and

$$
|g(\beta)|_{v} \leq \max \left\{1,|\beta|_{v}\right\}^{\operatorname{deg} g}|m|_{v}^{\operatorname{deg} g} .
$$

For $v \mid \infty$ and $|\beta|_{v}>1$, writing $|g(\beta)|_{v}=|\beta|_{v}^{\operatorname{deg} g}\left|g^{*}\left(\beta^{-1}\right)\right|_{v}$, where $g^{*}$ is the reciprocal of $g$, we have

$$
\begin{aligned}
|g(\beta)|_{v} & \leq \max \left\{1,|\beta|_{v}\right\}^{\operatorname{deg} g}\left(\sup _{|z| \leq 1} \max \left\{|g(z)|,\left|g^{*}(z)\right|\right\}\right)^{d_{v} / d} \\
& =\max \left\{1,|\beta|_{v}\right\}^{\operatorname{deg} g} \sup _{|z|=1}|g(z)|^{d_{v} / d}
\end{aligned}
$$

Hence assuming that $g(\beta) \neq 0$ we have

$$
1=\prod_{v}|g(\beta)|_{v} \leq H(\beta)^{\operatorname{deg} g} m^{-\operatorname{deg} g} \sup _{|z|=1}|g(z)|
$$

and

$$
h(\beta) \geq \log (m)-\frac{\log (\sqrt{M})}{\operatorname{deg} g}, \quad M:=\sup _{|z|=1}|g(z)|^{2} .
$$

It remains to check that $g(\beta) \neq 0$. By assumption $\beta \neq 1$. For $m$ odd $g_{1}\left(z^{n+1}\right)$ is irreducible by Lemma 2.2 so cannot vanish at $\alpha$ (which has degree at most $n$ ). From (3.1) we know that

$$
\prod_{v \mid \infty}|1-\beta|_{v} \geq m \prod_{v \nmid \infty} \max \left\{1,|\beta|_{v}\right\}^{-1} .
$$

So for $m>2$ we must have $\beta \neq-1$ (else (3.7) gives $2 \geq m$ ). Hence $g_{0}(\beta) g_{1}(\beta) \neq 0$. Thus when $s_{2}=0$ and $s_{1}=1$ (and $s_{0} \leq m^{2}-1$ when $m$ is odd), Lemma 2.1 gives

$$
\sqrt{M}=\frac{m^{s_{0}+1}}{\left(s_{0}+1\right)^{\frac{1}{2}\left(s_{0}+1\right)}}\left(\frac{4 s_{0}}{m^{2}-\delta}\right)^{\frac{1}{2} s_{0}},
$$

and

$$
H(\beta)^{s_{0}+1} \geq\left(s_{0}+1\right)^{\frac{1}{2}\left(s_{0}+1\right)}\left(\frac{m^{2}-\delta}{4 s_{0}}\right)^{\frac{1}{2} s_{0}} .
$$

The result (1.2)

$$
h(\beta) \geq \frac{1}{2} \log \left(\frac{m^{2}+4-\delta}{4}\right)
$$

follows from optimally taking $s_{0}=\left(m^{2}-\delta\right) / 4$.

Similarly, degree considerations show that $g_{2}(\beta) \neq 0$ when $4 \mid m$, or when $m$ is odd with $3 \nmid m$, and $g_{2}\left(z^{n+1}\right)$ is irreducible by Lemma 2.2. When $m$ is odd and $3 \mid m$, or when $2 \| m$, then $g_{2}(\beta) \neq 0$ from (2.3) and the lower bound (3.8). 
Converting to cosines, we have

$$
M=\sup _{|z|=1}|g(z)|^{2}=\sup _{u \in[-1,1]} \prod_{i=0}^{I} f_{i}(u)^{s_{i}}
$$

with

$$
\begin{gathered}
f_{0}(u)=2(1-u), \\
f_{1}(u)=\frac{1}{2}\left(m^{2}-\delta\right) u+\frac{1}{2}\left(m^{2}+\delta\right),
\end{gathered}
$$

and

$$
f_{2}(u)=\left(\frac{1}{2}\left(m^{2}+(4-\delta)\right) u+\frac{1}{2}\left(m^{2}-(4-\delta)\right)\right)^{2}
$$

where plainly $M$ will be achieved at $u=-1$ or at zero of

$$
\sum_{i=0}^{I} s_{i} \frac{f_{i}^{\prime}(u)}{f_{i}(u)}=0
$$

For example, after numerical computational and experimentation, the respective choices $\left(m ; s_{0}, s_{1}, s_{2}\right)=(3 ; 107,48,17),(5 ; 198,26,13)$, $(6 ; 246,21,11),(7 ; 225,14,8),(8 ; 151,7,4),(9 ; 326,12,7),(10 ; 106,3,2)$, and $(11 ; 206,5,3)$ produce in turn $c_{3}=0.599206, c_{5}=1.001086, c_{6}=1.172140$, $c_{7}=1.298988, c_{8}=1.429512, c_{9}=1.532875, c_{10}=1.637694$, and $c_{11}=$ 1.724309 .

For the asymptotic bound, we take a sequence of triples $\left(s_{0}, s_{1}, s_{2}\right)$ with

$$
s_{0} / s_{2} \rightarrow A m^{2}, \quad s_{1} / s_{2} \rightarrow 2 C,
$$

for constants $A$ and $C$ which will be chosen optimally below. Hence $M$ must be achieved at

$$
u=\frac{-A m^{6}+m^{2}((4-\delta)(2 C-A \delta)-2 \delta)-2 \delta(4-\delta)(C+1) \pm 2 m^{2} \sqrt{D_{1}}}{\left(m^{2}-\delta\right)\left(m^{2}+4-\delta\right)\left(A m^{2}+2 C+2\right)}
$$

where

$$
\begin{aligned}
D_{1}=m^{4} & \left((2 A+1+C)^{2}-8 A C\right) \\
& +m^{2}((2 A+1+C)(8-2 \delta(C+1))+8 A C \delta) \\
& +(4-\delta(1+C))^{2},
\end{aligned}
$$

or at $u=-1$ when $m$ is odd. Writing

$$
u=-1+\frac{2}{A m^{2}}(2 A+1+C-A \delta \pm \sqrt{D})+O\left(\frac{1}{m^{4}}\right)
$$


where $D=(2 A+1+C)^{2}-8 A C$, leads to

$$
\begin{aligned}
c_{m} \geq \log \left(\frac{m}{2}\right)+\frac{1}{2 A m^{2}} \min _{ \pm} \log \left(\frac{\exp (2 A+1+C-A \delta \pm \sqrt{D})}{\left(\frac{2 A+1+C \pm \sqrt{D}}{4 A}\right)^{2 C}\left(\frac{-2 A+1+C \pm \sqrt{D}}{4 A}\right)^{2}}\right) \\
\quad+O\left(\frac{1}{m^{4}}\right),
\end{aligned}
$$

or $\log (m / 2)+\frac{1}{A m^{2}} \log \left(2^{2(1+C)} / 3\right)+O\left(m^{-4}\right)$ if this is smaller when $m$ is odd. For a given choice of $C$ we can choose $A$ to make these \pm quantities equal. Choosing (after numerical experimentation) $2 C=1.5799148239$ and calculating $A=0.5569260220 \ldots$ gives the desired asymptotic bound.

To obtain the improved values for $m=3$ to 11 stated in the theorem, we take $g(z)=\prod_{i=0}^{I} g_{i}(z)^{s_{i}}$ with $I=4$ or 5 , where the auxiliary factors $g_{i}(z)$ and choice of exponents $s_{i}$ are given in Table 3.1. For these $g_{j}(z)$ we have $\left|g_{j}(\beta)\right|_{v} \leq|m|_{v}^{\operatorname{deg} g_{j}} \max \left\{1,|\beta|_{v}\right\}^{\operatorname{deg} g_{j}}$ for $v \nmid \infty$ and (3.6) holds as before (as long as $g(\beta) \neq 0$ ). We can argue as above that $g(\beta) \neq 0$ by irreducibility (and for $m=8$ that $\frac{1}{2} \log 9=1.0986 \ldots<1.4295 \ldots$, the previous lower bound, and for $m=5$ and $m=11$ that $\frac{1}{2} \log 8>1.016628$ and $\left.\frac{1}{2} \log 32>1.728890\right)$.

We remark that many factors of the auxiliary polynomials employed in the proof were selected by using a number of experimental strategies, including testing various combinations of factors of the form (3.3) or (3.4), since the polynomials in these families produce sizable arithmetic contributions to the bound (3.5) relative to their degree. Algorithm 2.3 of [2] was also used to construct some of the factors. For example, the polynomial $g_{3}(z)$ shown for $m=3$ in Table 3.1 was found by applying that algorithm to the base polynomial $(x-1)^{6}(x+2)^{3}\left(x^{2}+x+1\right)$. In addition, the values of the exponents $s_{i}$ used here were selected by using heuristic optimization strategies like hill-climbing.

We remark also that additional factors could probably be added to the auxiliary polynomials $g(z)$ employed here in the style of [2] for further improvements.

Finally, the choices $g(z)=\left(z^{2}-1\right)^{4}\left(z^{2}+1\right)$ and $g(z)=(z-1)^{m^{2}}(z+1)$ similarly recover the values $c_{2}=\frac{1}{4} \log 5$ and $c_{m}=\log \left(\sqrt{m^{2}+1} / 2\right)$ for $m>2$ respectively (and using the auxiliary polynomials of [2] for $g(z)$ gives the improved values stated there).

\section{Proof of Theorem 1.2}

Since the golden ratio is a limit point of Salem numbers with Littlewood minimal polynomials (Theorem 6.2 of [1]) we note that the optimal $c_{2}$ certainly satisfies (1.3). 
TABlE 3.1. Auxiliary factors and exponents.

\begin{tabular}{|c|c|c|}
\hline$m$ & Auxiliary factors $g_{3}(z), \ldots$ & $\left(s_{0}, s_{1}, s_{2}, s_{3}, \ldots\right)$ \\
\hline 3 & $\begin{array}{l}g_{3}(z)=11(z-1)^{4}+7 \cdot 3^{2} z(z-1)^{2}+3^{4} z^{2} \\
g_{4}(z)=13(z-1)^{4}+8 \cdot 3^{2} z(z-1)^{2}+3^{4} z^{2} \\
g_{5}(z)=5(z-1)^{2}+2 \cdot 3^{2} z\end{array}$ & $(823,178,183,48,53,7)$ \\
\hline 5 & $\begin{array}{l}g_{3}(z)=8(z-1)^{2}+5^{2} z \\
g_{4}(z)=61(z-1)^{4}+16 \cdot 5^{2} z(z-1)^{2}+5^{4} z^{2} \\
g_{5}(z)=5\left(11(z-1)^{4}+3 \cdot 5^{2} z(z-1)^{2}+5^{3} z^{2}\right)\end{array}$ & $(340,10,29,1,8,10)$ \\
\hline 6 & $\begin{array}{l}g_{3}(z)=109(z-1)^{4}+21 \cdot 6^{2} z(z-1)^{2}+6^{4} z^{2} \\
g_{4}(z)=11(z-1)^{2}+6^{2} z \\
g_{5}(z)=2\left(59(z-1)^{4}+11 \cdot 6^{2} z(z-1)^{2}+3 \cdot 6^{3} z^{2}\right)\end{array}$ & $\begin{array}{c}(222680,19000,8000 \\
2793,2064,1000)\end{array}$ \\
\hline 7 & $\begin{array}{l}g_{3}(z)=181(z-1)^{4}+27 \cdot 7^{2} z(z-1)^{2}+7^{4} z^{2} \\
g_{4}(z)=193(z-1)^{4}+28 \cdot 7^{2} z(z-1)^{2}+7^{4} z^{2} \\
g_{5}(z)=7\left(2 z^{2}+3 z+2\right)\end{array}$ & $(309,16,9,4,1,2)$ \\
\hline 8 & $\begin{array}{l}g_{3}(z)=2\left(9(z-1)^{2}+2^{5} z\right) \\
g_{4}(z)=305(z-1)^{4}+35 \cdot 8^{2} z(z-1)^{2}+8^{4} z^{2} \\
g_{5}(z)=321(z-1)^{4}+36 \cdot 8^{2} z(z-1)^{2}+8^{4} z^{2}\end{array}$ & $(944,45,20,5,5,2)$ \\
\hline 9 & $\begin{array}{l}g_{3}(z)=461(z-1)^{4}+43 \cdot 9^{2} z(z-1)^{2}+9^{4} z^{2} \\
g_{4}(z)=481(z-1)^{4}+44 \cdot 9^{2} z(z-1)^{2}+9^{4} z^{2}\end{array}$ & $(44277,0,1256,538,273)$ \\
\hline 10 & $\begin{array}{l}g_{3}(z)=701(z-1)^{4}+53 \cdot 10^{2} z(z-1)^{2}+10^{4} z^{2} \\
g_{4}(z)=1351(z-1)^{4}+104 \cdot 10^{2} z(z-1)^{2}+2 \cdot 10^{4} z^{2}\end{array}$ & $(1029,25,10,5,3)$ \\
\hline 11 & $\begin{array}{l}g_{3}(z)=32(z-1)^{2}+11^{2} z \\
g_{4}(z)=991(z-1)^{4}+63 \cdot 11^{2} z(z-1)^{2}+11^{4} z^{2} \\
g_{5}(z)=1021(z-1)^{4}+64 \cdot 11^{2} z(z-1)^{2}+11^{4} z^{2}\end{array}$ & $(827,6,12,2,6,3)$ \\
\hline
\end{tabular}

Suppose that $m \geq 3$. For (1.4) and (1.7) we take $n \geq 2$ and

$$
f_{n}(x)=x^{2 n}+\sum_{i=0}^{n-1}\left(x^{2 i}-(m-1) x^{2 i+1}\right)=\frac{x^{n+1}}{x^{2}-1} F_{n}(x),
$$

with

$$
F_{n}(x)=\left(x^{n+1}-x^{-(n+1)}\right)-(m-1)\left(x^{n}-x^{-n}\right) .
$$

Since $f_{n}\left(\frac{1}{m-1}\right)>0$ and $f_{n}\left(\frac{1}{m-1}\left(1+\left(\frac{2}{m-1}\right)^{n}\right)\right)<0$, it is clear that the $f_{n}(x)$ have real roots $\alpha_{n}$ and $\alpha_{n}^{-1}$ with $\alpha_{n} \rightarrow(m-1)$ as $n \rightarrow \infty$. Notice that $f_{n}(x)$ does not vanish at \pm 1 or any $(2 n+1)$ st root of unity (so by the theorem can have no cyclotomic factors). Since $\frac{1}{2 i} F\left(e^{2 \pi i t}\right)=$ $\sin (2 \pi(n+1) t)-(m-1) \sin (2 \pi n t)$ changes sign, it must have a zero $t_{j}$ in 
each interval $\left[\frac{2 j-1}{4 n}, \frac{2 j+1}{4 n}\right], j=1,2, \ldots, 2 n-1$, and the remaining $(2 n-2)$ zeros $e^{2 \pi i t_{j}}, t_{j} \neq 1 / 2$ of $f_{n}(x)$ all lie on the unit circle. Since $f_{n}(x)$ has no monic factors with all roots on the unit circle, these $f_{n}(x)$ are irreducible with $\left(\operatorname{deg} f_{n}+1\right) h\left(\alpha_{n}\right)=\left(\frac{2 n+1}{2 n}\right) \log \alpha_{n} \rightarrow \log (m-1)$ as $n \rightarrow \infty$.

For (1.5) we similarly consider

$$
f_{n, 4}(x)=\sum_{i=0}^{4 n+2} x^{i}-4 x \sum_{i=0}^{n} x^{4 i}=(1-x)\left(1-2 x-x^{2}\right) \sum_{i=0}^{n} x^{4 i}-x^{4 n+3}
$$

with real roots $\alpha_{n}, \alpha_{n}^{-1} \rightarrow \sqrt{2}-1, \sqrt{2}+1$ and no roots at the $(4 n+3)$ rd roots of unity. Writing $F_{n, 4}(x)=\left(x^{4}-1\right) f_{n, 4}(x) x^{-(2 n+3)}$, and observing that

$$
\frac{1}{4 i} F_{n, 4}\left(e^{2 \pi i t}\right)=(\cos (3 \pi t)+\cos (\pi t)) \sin ((4 n+3) \pi t)-2 \sin (4(n+1) \pi t)
$$

has sign changes in each of the intervals $[(2 j+1) / 8(n+1),(2 j+3) / 8(n+1)]$, $j=0, \ldots, 4 n+2$ (and removing the introduced fourth roots of unity), the remaining $4 n$ zeros of $f_{n, 4}(x)$ all lie on the unit circle.

For (1.6) we take

$$
\begin{aligned}
f_{n, 6}(x) & =\sum_{i=0}^{6 n+4} x^{i}-6 x\left(1-x+x^{2}\right) \sum_{i=0}^{n} x^{6 i} \\
& =(1-x)\left(1-x+x^{2}\right)\left(1-3 x-x^{2}\right) \sum_{i=0}^{n} x^{6 i}-x^{6 n+5}
\end{aligned}
$$

with real roots $\alpha_{n}, \alpha_{n}^{-1} \rightarrow \frac{1}{2}(\sqrt{13}-3), \frac{1}{2}(\sqrt{13}+3)$ and no roots at the $(6 n+5)$ th roots of unity. Writing $F_{n, 6}(x)=\frac{\left(x^{6}-1\right)}{\left(x^{2}-x+1\right)} f_{n, 6}(x) x^{-(3 n+4)}$ and observing that

$$
\frac{1}{4 i} F_{n, 6}\left(e^{2 \pi i t}\right)=(\cos (3 \pi t)+2 \cos (\pi t)) \sin ((6 n+5) \pi t)-3 \sin (6(n+1) \pi t)
$$

has sign changes in each of the intervals $[(2 j+1) / 12(n+1),(2 j+3) / 12(n+$ $1)], j=0, \ldots, 6 n+4$ (and removing the introduced sixth roots of unity), the remaining $6 n+2$ zeros of $f_{n, 6}(x)$ all lie on the unit circle.

\section{References}

[1] P. Borwein, E. Dobrowolski, and M. J. Mossinghoff, Lehmer's problem for polynomials with odd coefficients. Ann. of Math. (2) 166 (2007), no. 2, 347-366.

[2] A. Dubickas and M. J. Mossinghoff, Auxiliary polynomials for some problems regarding Mahler's measure. Acta Arith. 119 (2005), no. 1, 65-79.

[3] M. I. M. Ishak, M. J. Mossinghoff, C. G. Pinner, and B. Wiles, Lower bounds for heights in cyclotomic extensions. J. Number Theory 130 (2010), no. 6, 1408-1424.

[4] V. A. Lebesgue, Sur l'impossibilité, en nombres entiers, de l'équation $x^{m}=y^{2}+1$. Nouv. Ann. Math. (1) 9 (1850), 178-181.

[5] W. Ljungaren, Einige Bemerkungen über die Darstellung ganzer Zahlen durch binäre kubische Formen mit positiver Diskriminante. Acta Math. 75 (1943), 1-21. 
[6] T. Nagell, Des équations indéterminées $x^{2}+x+1=y^{n}$ et $x^{2}+x+1=3 y^{n}$. Norsk Matematisk Forening, Skr. Ser. I (1921), no. 2, 1-14.

J. GARZA

Department of Mathematics

Kansas State University

Manhattan, KS 66506

E-mail: johngarz@math.ksu.edu

M. I. M. ISHAK

Department of Mathematics

Kansas State University

Manhattan, KS 66506

E-mail: mimishak@math.ksu.edu

M. J. MossinghofF

Department of Mathematics

Davidson College

Davidson, NC 28035-6996

E-mail: mimossinghoff@davidson.edu

URL: http://www.davidson.edu/math/mossinghoff/

C. G. Pinner

Department of Mathematics

Kansas State University

Manhattan, KS 66506

E-mail: pinner@math.ksu.edu

B. WILES

Department of Mathematics

Kansas State University

Manhattan, KS 66506

E-mail: wilesb@math.ksu.edu 